\title{
Prognostic Factors Related to the Mortality Rate of Acute-on-Chronic Liver Failure Patients
}

\author{
Jian Zhang ${ }^{1,2, *}$ \\ Junfeng $\mathrm{Li}^{3, *}$ \\ Yu Chen ${ }^{1,2}$ \\ Mei Ding ${ }^{1,2}$ \\ Zhongping Duan ${ }^{1,2}$ \\ 'Difficult \& Complicated Liver diseases \\ and Artificial Liver Center, Beijing Youan \\ Hospital, Capital Medical University, \\ Beijing, People's Republic of China; \\ ${ }^{2}$ Beijing Municipal Key Laboratory of \\ Liver Failure and Artificial Liver \\ Treatment Research, Beijing, People's \\ Republic of China; ${ }^{3}$ The First Hospital of \\ Lanzhou University, Department of \\ Infectious Diseases, Lanzhou, People's \\ Republic of China
}

*These authors contributed equally to this work
Correspondence: Jian Zhang

Email zhangdajian1978@ccmu.edu.cn
Background: Critical illness-related corticosteroid insufficiency (CIRCI) is known to be a common complication in patients with acute-on-chronic liver failure (ACLF). However, factors that predict the survival rate of ACLF patients remain unclear. The present study aims to determine the prognostic factors that impinge on the survival rate of ACLF patients.

Methods: A total of 90 patients with ACLF at different stages, with or without CIRCI, were prospectively evaluated.

Results: Various clinical factors were found to be significantly different among patients at early, mid and late stages of ACLF, as well as between the same population of patients with and without CIRCI. Specifically, patients at later stages of ACLF and patients with CIRCI had significantly higher Child-Turcotte-Pugh (CTP), Model for End-Stage Liver Disease (MELD) and ACLF Research Consortium (AARC)-ACLF scores. CIRCI was observed in $20 \%$ of the enrolled patients (18 out of 90 ). In addition, the 90 -day mortality rate was higher in mid- and late-stage ACLF patients, as well as patients with CIRCI.

Conclusion: ACLF stage and CIRCI predict early mortality in patients with ACLF and could be actively monitored in these patients for prioritized liver transplantation.

Keywords: CIRCI, liver failure, mortality, stage

\section{Introduction}

Acute-on-chronic liver failure (ACLF) was first proposed by Dr. Ohnishi in 1995 to describe a group of acute injury symptoms established on top of chronic liver disease. ${ }^{1}$ Virus, bacterial and fungal infection, alcoholism and drug damage are all common causes of ACLF. Guidelines for Diagnosis and Treatment of Liver Failure (2018 Edition) published by the Chinese Society of Infectious Diseases proposed that ACLF is histopathologically manifested as the presence of newly identified liver cell necrosis at varying degrees on the basis of chronic liver failure. ${ }^{2}$ ACLF develops on top of chronic liver diseases due to various reasons, including acute jaundice deepening and coagulation dysfunction as syndromes manifested by liver failure. Other associated complications include hepatic encephalopathy, ascites, electrolyte imbalance, infection, hepatorenal syndrome, hepatopulmonary syndrome, and failure of extrahepatic organs. ACLF often progresses rapidly, with high short-term mortality rate and poor prognosis. So far, liver transplantation is the only effective treatment against ALCF. However, its clinical application is often limited due to the lack of liver source and high treatment costs. Therefore, prognosis of ACLF becomes extremely important to prevent the disease progression at early stage.

Due to the complicated conditions of liver failure, single indicators are not sufficient for prognosis and often cannot fully reflect liver function. Currently, the 
commonly used clinical prognostic scores include ChildTurcotte-Pugh (CTP), ${ }^{3}$ Model for End-Stage Liver Disease (MELD), ${ }^{4}$ MELD-Na ${ }^{5}$ and CLIF-SOFA. ${ }^{6}$ In 2017, the Asian Pacific Association for the Study of the Liver (APASL) established the ACLF Research Consortium (AARC)-ACLF score system that was superior to the previous ones in predicting mortality based on Asian population. ${ }^{7}$ It was proposed based on the total bilirubin, creatinine, serum lactate, INR and hepatic encephalopathy levels that were identified as independent predictors of mortality in ACLF patients. ${ }^{7}$

Relative adrenal insufficiency (RAI) is a condition characterized by the failure of the adrenal gland to produce sufficient amounts of steroid hormones, primarily cortisol, to meet the peripheral requirements. It is first observed in critically ill cirrhotic patients in $1960{ }^{8}$ Later studies have shown that RAI is closely associated with the onset of cirrhosis. $^{9-11}$ In addition, it is tightly correlated with compromised liver function and sharply elevated short-term mortality. ${ }^{12-14}$ The term RAI is later replaced by critical illness-related corticosteroid insufficiency (CIRCI), which is initially proposed by the Society of Critical Care Medicine in 2008 to describe disruption of the hypothalamic-pituitary-adrenal (HPA) axis during critical illness. ${ }^{15}$

In the present prospective cohort study, we aim to identify the prognostic factors that are related to the survival of ACLF patients. Specifically, our question is whether the presence of CIRCI, regardless of the stage of liver dysfunction, is associated with short-term mortality.

\section{Methods}

\section{Patients}

The study was approved by the review board of Beijing Youan Hospital of Capital Medical University. Verbal consent was obtained from all included patients or their relatives. Verbal informed consent was approved by the ethical committee, and the study was conducted in accordance with the Declaration of Helsinki. The present prospective cohort study enrolled 105 patients with ACLF at Beijing Youan Hospital from May 2019 to October 2020. ACLF was diagnosed according to clinical manifestation, laboratory data and compatible abdominal imaging criteria.

The stage of ACLF was determined as previously described. ${ }^{2}$ Specifically, early-stage ACLF develops on the basis of chronic non-cirrhotic liver disease. Patients at early ACLF stage exhibited extreme fatigue and severe gastrointestinal symptoms, such as anorexia, vomiting and abdominal distension. Serum total bilirubin was found to be over $10 \mathrm{mg} / \mathrm{dL}$ or with a daily increase over $1 \mathrm{mg} / \mathrm{dL}$. Patients demonstrated bleeding tendency, with prothrombin time (PT) (international normalized ratio (INR)) value between 1.5 and 1.9. No other complications or extrahepatic organ failure were observed.

The mid-stage ACLF develops on the basis of compensated liver cirrhosis, normally within 4 weeks. Patients at mid-ACLF stage demonstrated obvious bleeding with bleeding spots or ecchymosis and the PT (INR) value was between 1.9 and 2.6. Patients also demonstrated one complication and/or extrahepatic organ failure.

Late-stage ACLF develops on the basis of decompensated liver cirrhosis. Patients at late ACLF stage severe bleeding with ecchymosis at the injection site and the PT (INR) value was greater than 2.6. Patients also showed more than two or more complications and/or extrahepatic organ failure.

All included patients were between the age of 40 and 70. None of the patients were alcoholics or drug abusers and demonstrated stable haemodynamic parameters. Patients with tumors and dysfunction originated from failure in other organs were excluded from the study. No patients were taking any cortisol-related medication during the study period. Chronic adrenal insufficiency was ruled out in these patients by physical examinations, CT scan and track of their medical history. As a result, 15 patients were excluded from the initial enrollment.

\section{Baseline Evaluation}

An initial clinical evaluation on the day of admission was performed on all included patients, including measurement of blood pressure, pulse and respiratory rate and body temperature, as well as abdominal CT scan, electrocardiogram, liver and kidney function tests, measurement of serum high-density lipoprotein (HDL) and urine test. Routine biochemistry, hematology and coagulation profiles were also performed on the day of admission. The severity of liver failure was graded by CTP, MELD and AARCACLF scores.

\section{Determination of $\mathrm{CIRCI}$}

CIRCI was determined using the short corticotropin stimulation test (SST) as previously described. ${ }^{16}$ Briefly, synthetic adrenocorticotropic hormone (ACTH) was delivered intravenously. A high dose ACTH stimulation test $(250 \mathrm{mg}$ ) was used for the diagnosis of adrenal insufficiency. Blood sample was collected and cortisol levels 
were assessed immediately before and 30 and $60 \mathrm{~min}$ after the administration of ACTH. CIRCI was determined if the peak serum cortisol level was less than $18 \mu \mathrm{g} / \mathrm{dl}$ or the delta fraction was less than $9 \mu \mathrm{g} / \mathrm{dl}$.

\section{Follow-Up}

All patients were followed for 90 days since the day of hospital admission. Telephone communication or interview at the outpatient department was conducted on 7 , $14,28,60$, and 90 days after diagnosis.

\section{Statistical Analysis}

Quantitative data were presented as median plus range and analyzed by the Mann-Whitney U or One-way ANOVA following post-hoc test. A $\mathrm{P}$ value of less than 0.05 was considered as statistically significant. Survival analysis was assessed by the Kaplan-Meier curve and compared by the Log rank test. Univariate analysis of baseline factors was performed to predict the 90-day mortality. Factors that were identified as statistically significant were entered into multivariate analysis by binary logistic regression with the backward conditional method for further analysis.

Statistical analysis was conducted using the GraphPad Prism software (GraphPad Software Inc.) and SPSS 16.0 software (SPSS Inc.).

\section{Results}

\section{Comparison of Patient Characteristics Among the Early, Mid and Late Liver Failure Stage Groups}

A total of 105 consecutive patients diagnosed with liver failure were admitted to our institution and screened for the enrollment into the present study. Eventually, 15 patients were excluded according to the exclusion criteria. The remaining 90 patients were separated into 3 groups based on the stage of ACLF, ie early, mid and late stages. The clinical characteristics of all included patients are listed in Table 1.

Of the 90 patients, $64 \%$ (58 out of 90 ) of them were male. A significant difference in age was observed among the 3 groups, where patients in the early-stage group were much younger than the ones in the other two groups (Table 1). Alcohol and HBV infection were found to be the major causes of liver failure for patients in all 3 groups (Table 1). In addition, serum aspartate aminotransferase (AST), alanine aminotransferase (ALT), bilirubin, albumin, hemoglobin, high-density lipoprotein (HDL) levels, platelet count,
CTP, MELD and AARC-ACLF scores were all significantly different among the 3 groups (Table 1). Since the stages of ACLF were determined based on the prothrombin time (PT), it was certainly significantly different among the three groups (Table 1). In terms of cortisol level, basal, peak or the difference between the two were not significantly different among the 3 groups (Table 1). Only 1 patient in the early stage was determined to suffer CIRCI, whereas 10 and 7 patients met the CIRCI standard in the mid- and late-stage groups, respectively (Table 1).

\section{Comparison of Characteristics Between Patients with and without $\mathrm{CIRCl}$}

Next, we regrouped the 90 patients into 2 groups based on the presence of CIRCI, ie patients with CIRCI and patients without CIRCI. CIRCI was diagnosed in 18 of the patients (20\%). By comparing the clinical characteristics between the two groups of patients, we found that serum AST, bilirubin, albumin, hemoglobin levels, CTP, MELD and AARC-ACLF scores, basal, peak and delta fraction of cortisol levels were all significantly different (Table 2). This suggests that onset of CIRCI is closely related to reduced liver function.

\section{Mortality and $\mathrm{CIRCI}$}

The patients were followed for 90 days upon institution admission. 36 patients have passed away during this followup period, where 22 of them (22 out of 72, 30.6\%) were from the CIRCI-negative group and the other 14 (14 out of $18,77.8 \%$ ) were from the CIRCI-positive group (Figure 1A). When looking from the stage-grouping perspective, 2 ( 2 out of $30,6.7 \%$ ) were from the early-stage group, 10 (10 out of $30,33.3 \%$ ) were from the mid-stage group and 24 (24 out of $30,80 \%$ ) were from the late-stage group (Figure 1B). It is worth noting that all survivors did not receive orthotopic liver transplantation. The major causes of death were progressive liver failure and sepsis for both grouping conditions (Tables 3 and 4). We also performed Kaplan-Meier curve analysis to compare patient survival between the CIRCInegative and CIRCI-positive groups, as well as among the early, mid and late-stage groups. A significant difference was observed in both comparisons (Figure 1C and D). Taken together, these data suggest the mortality risk was much higher in the ACLF patients with CIRCI than in those without CIRCI, and much higher in patients at late and mid stages of ACLF than in those at early stage. 
Table I Clinical Characteristics of the Patients at Early, Mid and Late Stages of ACLF

\begin{tabular}{|c|c|c|c|c|}
\hline \multirow{2}{*}{ Characteristic } & \multicolumn{4}{|l|}{$\operatorname{ACLF}(n=90)$} \\
\hline & Early Stage $(n=30)$ & Mid Stage $(n=30)$ & Late Stage $(n=30)$ & $P$ value \\
\hline Age (years) & $48(4 \mid-67)$ & $56(40-69)$ & $56(43-69)$ & 0.0289 \\
\hline \multicolumn{5}{|l|}{ Sex } \\
\hline Male & 17 (56.7\%) & $20(66.7 \%)$ & 21 (70\%) & \\
\hline Female & $13(43.3 \%)$ & $10(33.3 \%)$ & $9(30 \%)$ & \\
\hline \multicolumn{5}{|l|}{ Cause } \\
\hline HBV & II (36.7\%) & $9(30 \%)$ & $10(33.3 \%)$ & \\
\hline $\mathrm{HCV}$ & $3(10 \%)$ & $3(10 \%)$ & $2(6.7 \%)$ & \\
\hline $\mathrm{HBV}+\mathrm{HCV}$ & $2(6.7 \%)$ & $4(13.3 \%)$ & $2(6.7 \%)$ & \\
\hline Alcohol & $13(43.3 \%)$ & $14(46.7 \%)$ & $15(50 \%)$ & \\
\hline Other & I (3.3\%) & $0(0 \%)$ & I (3.3\%) & \\
\hline AST (U/L) & $43.3(27.1-54.4)$ & $53.35(40.1-68)$ & $67.6(55.8-83.9)$ & $P<0.000$ I \\
\hline ALT (U/L) & $22.5(15-29.7)$ & $33.7(21.1-39.7)$ & $43.2(30.1-49.8)$ & $P<0.0001$ \\
\hline Bilirubin (mg/dL) & $2.55(2-3)$ & $3.6(3-4.5)$ & $4.1(3.6-4.9)$ & $P<0.000 I$ \\
\hline Albumin $(g / d L)$ & $3.1(2.5-4)$ & $2.5(2-3.5)$ & $2.5(2-2.9)$ & $P<0.000$ I \\
\hline PT (INR) & 1.7 (1.5-1.9) & $2.4(2-2.6)$ & $2.9(2.7-3)$ & $P<0.000 I$ \\
\hline Leukocytes (xI09/L) & $4.75(1.7-7.7)$ & $4.65(1.7-7.6)$ & $4.35(1.8-7.6)$ & $P=0.9893$ \\
\hline Hemoglobin (g/dL) & $12.45(11-13)$ & $10.6(9-12)$ & $9.95(9-11)$ & $P<0.000 I$ \\
\hline Platelet count (x109/L) & $82.8(55.4-145.4)$ & II $4.55(46.7-144)$ & $87.75(47.9-145.5)$ & $\mathrm{P}=0.0225$ \\
\hline $\mathrm{Na}(\mathrm{mmol} / \mathrm{L})$ & $14 \mid .35(\mid 35.7-144.8)$ & $139.8(135-145)$ & | 40.55 (|35.7-|44.9) & $P=0.2663$ \\
\hline $\mathrm{K}(\mathrm{mmol} / \mathrm{L})$ & $4.2(3-4.9)$ & $4(3.2-4.9)$ & $4.25(3-5)$ & $P=0.6756$ \\
\hline BUN (mg/dL) & $13.75(9.5-16.9)$ & $12.55(8-17)$ & $12.9(8.2-16.9)$ & $P=0.5030$ \\
\hline Creatinine $(\mathrm{mg} / \mathrm{dL})$ & $0.8(0.5-1)$ & $0.8(0.5-1)$ & $0.7(0.5-1)$ & $P=0.6327$ \\
\hline $\mathrm{HDL}(\mathrm{mg} / \mathrm{dL})$ & $24.55(20-32.4)$ & $29.15(20.1-39.8)$ & $36.15(24.7-49.2)$ & $P<0.0001$ \\
\hline CTP score & $7(6-8)$ & $9(7-10)$ & $9(7-11)$ & $P<0.000 I$ \\
\hline \multicolumn{5}{|l|}{ CTP class } \\
\hline$A$ & $10(33.3 \%)$ & $0(0 \%)$ & $0(0 \%)$ & $P<0.000 I$ \\
\hline B & $20(66.7 \%)$ & $21(70 \%)$ & $18(60 \%)$ & $P<0.000 I$ \\
\hline C & $0(0 \%)$ & $9(30 \%)$ & $12(40 \%)$ & $P<0.000 I$ \\
\hline MELD score & $13(10-15)$ & $15.5(10-25)$ & $19.5(15-25)$ & $P<0.0001$ \\
\hline AARC-ACLF score & $6(5-8)$ & $10(7-11)$ & $12(9-15)$ & $P<0.0001$ \\
\hline Basal cortisol ( $\mu \mathrm{g} / \mathrm{dL})$ & $10.8(8.5-15)$ & $11.4(8.1-15.7)$ & $11.8(8.1-16)$ & $P=0.5|4|$ \\
\hline Peak cortisol ( $\mu \mathrm{g} / \mathrm{dL})$ & $26.1(19.7-32.4)$ & $23.7(17.1-39.3)$ & $24.9(15.5-34.3)$ & $P=0.9646$ \\
\hline Delta fraction cortisol ( $\mu \mathrm{g} / \mathrm{dL})$ & I5.6 (8-2|.4) & $\mid 2.2(4.4-3 \mid .2)$ & $13.5(5.6-22.3)$ & $P=0.8427$ \\
\hline $\mathrm{ClRCl}(\%)$ & I (3.3\%) & $10(33.3 \%)$ & $7(23.3 \%)$ & \\
\hline
\end{tabular}

Note: Data are presented as median (range) or actual number (percentage).

Abbreviations: HBV, hepatitis B virus; HCV, hepatitis C virus; AST, aspartate aminotransferase; ALT, alanine aminotransferase; PT, prothrombin time; BUN, blood urea nitrogen; HDL, high-density lipoprotein; CTP, Child-Turcotte-Pugh; MELD, Model For End-Stage Liver Disease; CIRCl, critical illness-related corticosteroid insufficiency; INR, international normalized ratio. 
Table 2 Clinical Characteristics of the ACLF Patients with and without CIRCI

\begin{tabular}{|c|c|c|c|}
\hline & Patients without CIRCI $(n=72)$ & Patients with $\mathrm{CIRCI}(\mathrm{n}=18)$ & $P$ value \\
\hline \multicolumn{4}{|l|}{ Characteristic } \\
\hline Age (years) & $52(4 \mid-69)$ & $56(40-69)$ & $P=0.4287$ \\
\hline AST (U/L) & $52.4(27.1-83.9)$ & $62.8(41.9-80.4)$ & $P=0.0162$ \\
\hline $\operatorname{ALT}(\mathrm{U} / \mathrm{L})$ & $31.4(15-49.8)$ & $34.3(22.3-49.3)$ & $P=0.1234$ \\
\hline Bilirubin (mg/dL) & $3.5(2-4.9)$ & $3.9(2.5-4.9)$ & $P=0.0194$ \\
\hline Albumin $(g / d L)$ & $2.8(2-4)$ & $2.3(2.2-3.3)$ & $P=0.0142$ \\
\hline PT (INR) & $2.3(1.5-3)$ & $2.6(1.6-3)$ & $P=0.0796$ \\
\hline Leukocytes (x109/L) & $4.5(1.7-7.7)$ & $5.1(1.8-7.2)$ & $\mathrm{P}=0.8842$ \\
\hline Hemoglobin (g/dL) & II (9-13) & $10.6(9-11.7)$ & $P=0.0300$ \\
\hline Platelet count (x109/L) & $93.5(46.7-145.4)$ & II $5.2(54 .|-| 45.5)$ & $P=0.0577$ \\
\hline $\mathrm{Na}(\mathrm{mmol} / \mathrm{L})$ & $140.9(\mid 35.5-144.9)$ & $139.4(135-145)$ & $P=0.1913$ \\
\hline $\mathrm{K}(\mathrm{mmol} / \mathrm{L})$ & $4.1(3-5)$ & $4(3.2-4.8)$ & $P=0.6468$ \\
\hline BUN (mg/dL) & $12.7(8-17)$ & $13.4(8.6-17)$ & $P=0.5344$ \\
\hline Creatinine $(\mathrm{mg} / \mathrm{dL})$ & $0.8(0.5-1)$ & $0.6(0.5-1)$ & $P=0.1022$ \\
\hline HDL (mg/dL) & $28.5(20-49.2)$ & $30.7(20.4-42.3)$ & $P=0.4787$ \\
\hline CTP score & $8(5.5-10.4)$ & $9.4(6-10.5)$ & $P=0.0075$ \\
\hline MELD score & $15(10-25)$ & $19(10-25)$ & $\mathrm{P}=0.0280$ \\
\hline AARC-ACLF score & $9(5-15)$ & $11(6-15)$ & $P=0.0322$ \\
\hline Basal cortisol ( $\mu \mathrm{g} / \mathrm{dL})$ & $10.9(8.1-16)$ & I3.3 (8.|-|5.7) & $P=0.0012$ \\
\hline Peak cortisol ( $\mu \mathrm{g} / \mathrm{dL})$ & $27.2(18.8-39.3)$ & $19.35(15.5-24.2)$ & $P<0.0001$ \\
\hline Delta fraction cortisol $(\mu \mathrm{g} / \mathrm{dL})$ & $15.9(8.6-3 \mid .2)$ & $6.3(4.4-8.7)$ & $P<0.0001$ \\
\hline
\end{tabular}

Note: Data are presented as median (range) or actual number (percentage).

Abbreviations: HBV, hepatitis B virus; HCV, hepatitis C virus; AST, aspartate aminotransferase; ALT, alanine aminotransferase; PT, prothrombin time; BUN, blood urea nitrogen; HDL, high-density lipoprotein; CTP, Child-Turcotte-Pugh; MELD, Model For End-Stage Liver Disease; CIRCI, critical illness-related corticosteroid insufficiency; INR, international normalized ratio.

Cox regression analysis of factors that predict the 90day survival rate revealed that serum albumin, hemoglobin and HDL levels, CTP, MELD and AARC-ACLF scores, as well as delta fraction cortisol level were significantly related factors in univariate analysis (Table 5).

\section{Discussion}

In the present study, we employed the peak serum cortisol level that is less than $18 \mu \mathrm{g} / \mathrm{dl}$ or the delta fraction that is less than $9 \mu \mathrm{g} / \mathrm{dl}$ as criteria to determine CIRCI in ACLF patients. With that, we revealed 20\% (18 out of 90) CIRCI in these patients at different stages. A number of methods have been developed to evaluate adrenal function, including assessing cortisol levels after insulin-induced hypoglycemia, or administration of ACTH, or corticotropin-releasing hormone. $^{15,17,18}$ The CIRCI frequency of $20 \%$ identified in this study falls within the previously published range of similar studies. ${ }^{13,14,19-21}$ In line with previous study, ${ }^{19}$ we found that CIRCI prevalence was tightly correlated with liver function as reflected by the CTP, MELD and AARC-ACLF scores. In addition, it was also dramatically elevated in ACLF patients at mid and late stages, compared to those at early stage. The factors that were found to be significantly worsened in CIRCI patients were generally consistent with previously published studies, ${ }^{19,20}$ where the only difference was that prothrombin time was not identified in the present study. On top, we also compared various factors among patients at early, mid and late stages of ACLF. More factors were found to be significantly different among the 3 groups as identified for the CIRCI comparison.

Several potential underlying mechanisms that correlate CIRCI with liver failure have been previously proposed, including 1) liver dysfunction that lowers serum cholesterol level and subsequent reduced cortisol biosynthesis, 2) excessive production of proinflammatory cytokines that suppresses adrenal steroid production and 3) circulatory dysfunction that leads to adrenal hypoperfusion. ${ }^{12,17,22}$ In general, our results agree with the first mechanism since CIRCI was tightly correlated with more advanced ACLF and lower serum HDL level. The finding that CIRCI patients had a significantly lower level of hemoglobin suggests that CIRCI might be associated with the circulatory dysfunction commonly observed in late-stage liver failure patients. Therefore, it is likely that the development of CIRCI in ACLF is a combination of different mechanisms, instead of a single factor. 

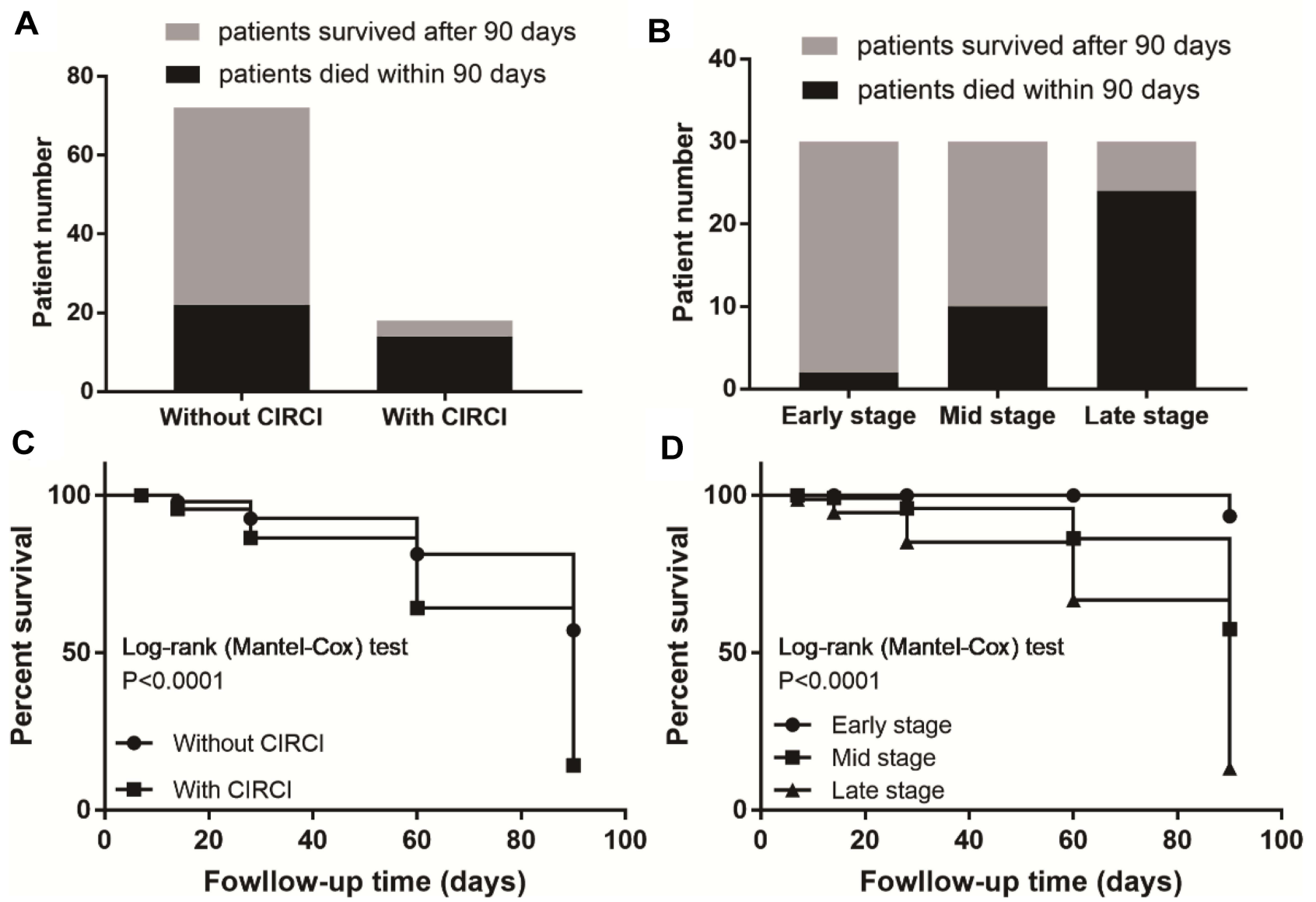

Figure I Survival analysis of patients at early, mid and late stages of ACLF, and patients with and without $\mathrm{CIRCI}$. (A and $\mathbf{B})$ Survival outcome of $\mathrm{CIRCI}$-negative and CIRCIpositive patients (A) and patients at early, mid and late stages of ACLF (B). (C and D) Kaplan-Meier curves showing survival of patients with and without CIRCI (C) and patients at early, mid and late stages of ACLF (D); and compared with the Log rank test.

Another important finding of the present study is the positive correlation between the stage of ACLF and 90day mortality, as well as CIRCI and 90-day mortality. Importantly, the presence of CIRCI is independently associated with the short-term mortality, regardless of the stage of liver dysfunction. To the best of our knowledge, our study is the first to investigate the impact of ACLF stage

Table 3 Cause of Death of the ACLF Patients with and without $\mathrm{CIRCl}$

\begin{tabular}{|c|c|c|}
\hline Cause of Death & $\begin{array}{l}\text { Patients Died } \\
\text { without CIRCI } \\
(n=22)\end{array}$ & $\begin{array}{l}\text { Patients Died } \\
\text { with CIRCI } \\
(n=\mid 4)\end{array}$ \\
\hline Progressive liver failure & $10(45.5 \%)$ & $6(42.9 \%)$ \\
\hline Sepsis & $6(27.3 \%)$ & $4(28.6 \%)$ \\
\hline Gastrointestinal bleeding & $2(9.1 \%)$ & $2(14.3 \%)$ \\
\hline Hepatorenal syndrome & $2(9.1 \%)$ & I (7.I\%) \\
\hline Hepatocellular carcinoma & $2(9.1 \%)$ & I (7.I\%) \\
\hline
\end{tabular}

Note: Data are presented as actual number (percentage). on mortality. The findings that CIRCI is associated much higher mortality rate are consistent with several previous studies. $^{12,19-21}$ Therefore, CIRCI appears to be a critical test for liver failure patients at different stages to direct further treatment and precautions.

There are a few limitations associated with the present study. First, the analyzed patient number was relatively

Table 4 Cause of Death of the Patients at Early, Mid and Late Stages of ACLF

\begin{tabular}{|l|l|l|l|}
\hline Cause of Death & $\begin{array}{l}\text { Early } \\
\text { Stage } \\
(\mathbf{n}=2)\end{array}$ & $\begin{array}{l}\text { Mid } \\
\text { Stage } \\
(\mathbf{n}=10)\end{array}$ & $\begin{array}{l}\text { Late } \\
\text { Stage } \\
(\mathbf{n}=\mathbf{2 4})\end{array}$ \\
\hline $\begin{array}{l}\text { Progressive liver failure } \\
\text { Sepsis }\end{array}$ & $\mathrm{I}(50 \%)$ & $5(50 \%)$ & $\mathrm{II}(45.8 \%)$ \\
Gastrointestinal bleeding & $\mathrm{I}(50 \%)$ & $2(20 \%)$ & $7(29.2 \%)$ \\
Hepatorenal syndrome & $0(0 \%)$ & $0(0 \%)$ & $2(8.3 \%)$ \\
Hepatocellular carcinoma & $0(0 \%)$ & $\mathrm{I}(10 \%)$ & $2(8.3 \%)$ \\
\hline
\end{tabular}

Note: Data are presented as actual number (percentage). 
Table 5 Cox Regression Univariate Analysis of Factors Predicting 90-Day Mortality

\begin{tabular}{|l|l|l|l|}
\hline Characteristic & HR & $\mathbf{9 5 \%} \mathbf{C l}$ & P value \\
\hline & Univariate analysis & & \\
Age & 1.032 & $0.913-1.098$ & $\mathrm{P}=0.75 \mathrm{I}$ \\
Male & 0.812 & $0.219-2.342$ & $\mathrm{P}=0.721$ \\
AST & 0.992 & $0.943-1.135$ & $\mathrm{P}=0.397$ \\
ALT & 0.917 & $0.945-1.178$ & $\mathrm{P}=0.362$ \\
Bilirubin & 1.204 & $0.931-1.465$ & $\mathrm{P}=0.169$ \\
Albumin & 0.053 & $0.022-0.479$ & $\mathrm{P}=0.004$ \\
PT (INR) & 6.332 & $2.467-15.963$ & $\mathrm{P}=0.078$ \\
Leucocytes & 1.021 & $0.954-1.089$ & $\mathrm{P}=0.384$ \\
Hemoglobin & 0.714 & $0.457-0.986$ & $\mathrm{P}=0.022$ \\
Platelet count & 1.004 & $0.941-1.187$ & $\mathrm{P}=0.379$ \\
Na & 0.941 & $0.798-1.096$ & $\mathrm{P}=0.642$ \\
K & 0.865 & $0.694-0.973$ & $\mathrm{P}=0.514$ \\
BUN & 0.798 & $0.675-1.085$ & $\mathrm{P}=0.473$ \\
Creatinine & 9.765 & $2.81-18.645$ & $\mathrm{P}=0.097$ \\
HDL & 0.978 & $0.896-0.998$ & $\mathrm{P}=0.012$ \\
CTP score & 1.573 & $1.153-2.123$ & $\mathrm{P}=0.002$ \\
MELD score & 1.187 & $1.021-1.375$ & $\mathrm{P}=0.008$ \\
AARC-ACLF score & 1.346 & $1.045-1.875$ & $\mathrm{P}=0.003$ \\
Delta fraction cortisol & 0.824 & $0.692-0.948$ & $\mathrm{P}=0.001$ \\
\hline
\end{tabular}

Abbreviations: HBV, hepatitis B virus; HCV, hepatitis C virus; AST, aspartate aminotransferase; ALT, alanine aminotransferase; PT, prothrombin time; BUN, blood urea nitrogen; HDL, high-density lipoprotein; CTP, Child-Turcotte-Pugh; MELD, Model For End-Stage Liver Disease; INR, international normalized ratio.

limited, due to the nature of this single-center study. Future studies involving multiple centers with larger patient numbers would be needed to strengthen the findings. Second, we did not manage to assess direct hemodynamic parameters and serum cytokine levels to test the involvement of inflammation mechanisms on adrenal production of steroids and its relation to CIRCI and liver failure. Third, due to increased sex hormone-binding globulin (SHBG) under liver dysfunction conditions, free cortisol might be normal even in the presence of low total cortisol. Therefore, further study measuring free urine cortisol level in these patients may solidify the conclusion of the current study. Fourth, the follow-up period is relatively short, although an overall $40 \%$ mortality rate (36 out of 90) were reached after 90 days of follow-up.

In summary, we have identified a number of clinical factors that were significantly different in patients at different stages of ACLF, as well as in the patients with or without CIRCI. Our data demonstrate that the prevalence of CIRCI is positively correlated with the severity of ACLF. In addition, both ACLF stages and onset of CIRCI are important predictors for the 90-day mortality rate.

\section{Abbreviations}

HBV, hepatitis B virus; HCV, hepatitis C virus; AST, aspartate aminotransferase; ALT, alanine aminotransferase; PT, prothrombin time; BUN, blood urea nitrogen; HDL, high-density lipoprotein; CTP, Child-TurcottePugh; MELD, Model For End-Stage Liver Disease; RAI, relative adrenal insufficiency; INR, international normalized ratio.

\section{Data Sharing Statement}

All data generated or analyzed during this study are included in this published article.

\section{Ethics Approval and Consent to Participate}

The study protocol was approved by Beijing Youan Hospital of Capital Medical University. Verbal consent was obtained from each patient at the time of admission. The purpose and design of the study were carefully explained to all individual participants accompanied by their close relatives before consent was obtained according to the guidance provided by the ethical committee. The patients provided their written informed consent to participate in this study.

\section{Consent for Publication}

All authors read and approved the final manuscript.

\section{Acknowledgment}

The study was supported by Beijing Key Lab Open Project Funding (BJYAHKF2017007).

\section{Author Contributions}

All authors made substantial contributions to conception and design, acquisition of data, or analysis and interpretation of data; took part in drafting the article or revising it critically for important intellectual content; agreed to submit to the current journal; gave final approval of the version to be published; and agreed to be accountable for all aspects of the work.

\section{Funding}

Beijing Key Lab Open Project Funding (BJYAHKF2 017007).

\section{Disclosure}

The authors declare that they have no competing interests. 


\section{References}

1. Ohnishi H, Sugihara J, Moriwaki H, et al. [Acute-on-chronic liver failure]. Ryoikibetsu Shokogun Shirizu. 1995;(7):217-219. [Indonesian]

2. Liver Failure and Artificial Liver Goup, Chinese Society of Infectious Diseases, Chinese Medical Association, Severe Liver Disease and Artificial Liver Group, Chinese Society of Hepatology, Chinese Medical Association. Guidelines for diagnosis and treatment of liver failure (2018 Edition). Zhong Hua Chuan Ran Bing Za Zhi. 2019;37:1-9.

3. Pugh RN, Murray-Lyon M, D. J, et al. Transection of the oesophagus for bleeding oesophageal varices. Br J Surg. 1973;60(8):646-649. doi:10.1002/bjs. 1800600817

4. Malinchoc M, Kamath PS, Gordon FD, et al. A model to predict poor survival in patients undergoing transjugular intrahepatic portosystemic shunts. Hepatology. 2000;31(4):p. 864-71. doi:10.1053/he.2000.5852

5. Biggins SW, Kim WR, Terrault NA, et al. Evidence-based incorporation of serum sodium concentration into MELD. Gastroenterology. 2006;130(6):p. 1652-1660. doi:10.1053/j.gastro.2006.02.010

6. Moreau R, Jalan R, Gines P, et al. Acute-on-chronic liver failure is a distinct syndrome that develops in patients with acute decompensation of cirrhosis. Gastroenterology. 2013;144(7):p. 1426-U189. doi:10.1053/j.gastro.2013.02.042

7. Choudhury A, Jindal A, Maiwall R, et al. Liver failure determines the outcome in patients of acute-on-chronic liver failure (ACLF): comparison of APASL ACLF research consortium (AARC) and CLIF-SOFA models. Hepatol Int. 2017;11(5):p. 461-471. doi:10.1007/s12072-017-9816-Z

8. Peterson RE. Adrenocortical steroid metabolism and adrenal cortical function in liver disease. J Clin Invest. 1960;39(2):320-331. doi:10.1172/JCI104043

9. Harry R, Auzinger G, Wendon J. The clinical importance of adrenal insufficiency in acute hepatic dysfunction. Hepatology. 2002;36(2): p. $395-402$.

10. McDonald JA, Handelsman DJ, Dilworth P, et al. Hypothalamicpituitary adrenal function in end-stage non-alcoholic liver disease. $J$ Gastroenterol Hepatol. 1993;8(3):p. 247-53. doi:10.1111/j.14401746.1993.tb01195.x

11. Tsai MH, Peng Y-S, Chen Y-C, et al. Adrenal insufficiency in patients with cirrhosis, severe sepsis and septic shock. Hepatology. 2006;43 (4):p. 673-81. doi:10.1002/hep.21101

12. Acevedo J, Fernández J, Prado V, et al. Relative adrenal insufficiency in decompensated cirrhosis: relationship to short-term risk of severe sepsis, hepatorenal syndrome, and death. Hepatology. 2013;58(5):p. 1757-65. doi:10.1002/hep.26535
13. Fede G, Spadaro L, Tomaselli T, et al. Assessment of adrenocortical reserve in stable patients with cirrhosis. $J$ Hepatol. 2011;54(2):p. 243-50. doi:10.1016/j.jhep.2010.06.034

14. Thevenot T, Borot S, Remy-Martin A, et al. Assessment of adrenal function in cirrhotic patients using concentration of serum-free and salivary cortisol. Liver Int. 2011;31(3):p. 425-33. doi:10.1111/j.14783231.2010.02431.x

15. Marik PE, McDONALD JA, Handelsman DJ, et al. Recommendations for the diagnosis and management of corticosteroid insufficiency in critically ill adult patients: consensus statements from an international task force by the American College of Critical Care Medicine. Crit Care Med. 2008;36(6):p. 1937-49. doi:10.1097/ CCM.0b013e31817603ba

16. Huang XF, Hu W, He XJ, Zhang GS. A potential diagnostic protocol for critical illness-related corticosteroid insufficiency (CIRCI) in critically ill patients. J Eur Crit Care Med. 2018;2:86. doi:10.21037/ jeccm.2018.10.11

17. Fede G, Spadaro L, Tomaselli T, et al. Adrenocortical dysfunction in liver disease: a systematic review. Hepatology. 2012;55(4):p. 1282-91. doi: 10.1002/hep. 25573

18. Kazlauskaite R, Evans AT, Villabona CV, et al. Corticotropin tests for hypothalamic-pituitary- adrenal insufficiency: a metaanalysis. J Clin Endocrinol Metab. 2008;93(11):p. 4245-53. doi:10.1210/jc.20080710

19. Chawlani R, A Arora, P Ranjan, et al. Adrenal insufficiency predicts early mortality in patients with cirrhosis. United European Gastroenterol J. 2015;3(6):p. 529-38. doi:10.1177/205064061455 2314

20. Jang JY, Kim TY, Sohn JH, et al. Relative adrenal insufficiency in chronic liver disease: its prevalence and effects on long-term mortality. Aliment Pharmacol Ther. 2014;40(7):p. 819-26. doi:10. 1111/apt.12891

21. Tan T, Chang L, Woodward A, et al. Characterising adrenal function using directly measured plasma free cortisol in stable severe liver disease. J Hepatol. 2010;53(5):p. 841-8. doi:10.1016/j.jhep.2010. 05.020

22. Fernandez J, A Escorsell, M Zabalza,et al. Adrenal insufficiency in patients with cirrhosis and septic shock: effect of treatment with hydrocortisone on survival. Hepatology. 2006;44(5):p. 1288-95.

Diabetes, Metabolic Syndrome and Obesity: Targets and Therapy

\section{Publish your work in this journal}

Diabetes, Metabolic Syndrome and Obesity: Targets and Therapy is an international, peer-reviewed open-access journal committed to the rapid publication of the latest laboratory and clinical findings in the fields of diabetes, metabolic syndrome and obesity research. Original research, review, case reports, hypothesis formation, expert opinion and commentaries are all considered for publication. The manuscript management system is completely online and includes a very quick and fair peer-review system, which is all easy to use. Visit http://www.dovepress.com/testimonials.php to read real quotes from published authors. 